\title{
The perceived position of the hand in space
}

\author{
PATRICK HAGGARD, CHRIS NEWMAN, JUSTINE BLUNDELL, and HOLLY ANDREW \\ University College London, London, England
}

\begin{abstract}
This paper reports a series of experiments of the perceived position of the hand in egocentric space. The experiments focused on the bias in the proprioceptively perceived position of the hand at a series of locations spanning the midline from left to right. Perceived position was tested in a matching paradigm, in which subjects indicated the perceived position of a target, which could have been either a visual stimulus or their own fingertip, by placing the index finger of the other hand in the corresponding location on the other side of a fixed surface. Both the constant error, or bias, and the variable error, or consistency of matching attempts, were measured. Experiment 1 showed that (1) there is a far-left advantage in matching tasks, such that errors in perceived position are significantly lower in extreme-left positions than in extreme-right positions, and (2) there is a strong hand-bias effect in the absence of vision, such that the perceived positions of the left and right index fingertips held in the same actual target position in fact differ significantly. Experiments 2 and 3 demonstrated that this hand-bias effect is genuinely due to errors in the perceived position of the matched hand, and not to the attempt at matching it with the other hand. These results suggest that there is no unifying representation of egocentric, proprioceptive space. Rather, separate representations appear to be maintained for each effector. The bias of these representations may reflect the motor function of that effector.
\end{abstract}

The representation of space in the nervous system has been a central topic in modern neuroscience and in psychology (Paillard, 1991). In the human literature, much interest has focused on distortions of spatial representation, whereby the perceived positions of objects do not correspond to their actual positions. Much of this literature has been driven by the topic of spatial neglect, in which the left side of space appears to be lost, compressed, distorted, or attenuated following lesions of the right posterior parietal cortex (Bisiach, 1993). Studies of neglect have led several researchers to try to identify the basic organization of normal egocentric space. For example, the body midline has been held to define a primary axis of egocentric space (Jeannerod, 1988). In fact, several studies of normal human performance point to small distortions in the perception of left-right location in egocentric space, reminiscent of the more dramatic effects seen in patients with left neglect. Thus, Pierson-Savage and Bradshaw (1987) have described a far-left disadvantage, whereby stimuli presented in extreme-left space appear to be processed less efficiently than other stimuli. However, since the processing required in their task (speeded responses to a vibrotactile stimulation to the tip of the index finger positioned at various points along the left-right axis) was not intrinsically spatial, their data cannot conclusively show that far-left disadvantage is due to impoverished spatial representation of the far left of egocentric space.

In normals, some studies have focused on the purely egocentric reference, by asking subjects to indicate the sub-

Correspondence should be addressed to P. Haggard, Department of Psychology, University College London, Gower St., London WC1E 6BT, England (e-mail: p.haggard@ucl.ac.uk).

-Accepted by previous editor, Myron L. Braunstein jective midline by pointing straight ahead of them. However, whereas Werner, Wapner, and Bruell (1953) found a rightward bias in the perceived location of the midline in the absence of visual cues, Biguer and Jeannerod (reported in Jeannerod, 1988) reported a bias in the opposite leftward direction using a similar paradigm. Another tradition has investigated representations of external locations using egocentric encoding of a range of external positions. Thus, a left-side underestimation in line bisection tasks (Bradshaw, Nettleton, Nathan, \& Wilson, 1983) suggests variations in spatial processing along the leftright dimension. However, this bias has not been found when the task is performed in left hemispace (Bowers \& Heilman, 1980), suggesting there may not be a ubiquitous bias in the perception of egocentric space.

A more specific question about egocentric spatial representation can be asked by investigating distortions in the perceived position of the hand at several points along the left-right dimension, not just at the midline. The nervous system has been thought to maintain a body schema (Head, 1920) - an integrated supramodal representation of the layout of the body parts in space. More recently, Ghilardi, Gordon, and Ghez (1995) have reported an interesting distortion of the proprioceptively perceived position of the hand. They found that subjects consistently underestimate the distance of the hand from the perceptual midline. This distortion was inferred from a systematic directional bias in pointing movements. However, their study focused on directional effects on hand paths of reaching movements and did not explicitly map the perceived position of the hand at a range of locations along the left-right axis.

Potentially the most powerful methodology for studying the perceived position of the hand in space is the matching technique (von Hofsten \& Rosblad, 1988; Wann, 1991). 
This involves presenting the subject with a target location on a solid surface. The target location may be visual (a mark on the surface), proprioceptive (placing the subject's fingertip on a specific location on the surface), or both (the subject sees the fingertip in the target location on the surface). The perceived position of the target is then assessed by asking the subject to place the index finger on the other side of the surface in the position that the target is perceived to occupy on the original side. If one hand is already being used to mark the target, as in proprioceptive conditions, the other hand is used to match it on the other side of the surface. Typically, the match will be made without the benefit of vision, since subjects cannot see the hand moving below the surface. The main interest of previous studies has been in the differences between three matching conditions: visual:proprioceptive $(\mathrm{V}: \mathrm{P})$, visual and proprioceptive:proprioceptive (VP:P), and proprioceptive:proprioceptive (P:P). The difference between V:P and $P: P$ performance can be attributed to the relatively low acuity of the proprioceptive system in comparison with vision. Both Wann (1991) and Von Hofsten and Rosblad (1988) compared the accuracy of matching in the three conditions. Both studies found that the VP:P condition produced the smallest matching errors, followed by the V:P condition. Performance in the P:P condition was the worst. These results were interpreted as evidence for sensorimotor integration, whereby a combined visual and proprioceptive representation of location in egocentric space is superior to a representation using just one of those modalities. Van Beers, Sittig, and Denier van der Gon (1996) found that the variable error of performance in a VP:P condition was smaller than expected from the variances of V:P and $P: P$ performance, again suggesting integration. Additional studies (Baud-Bovy \& Viviani, 1998; Helms-Tillery, Flanders, \& Soechting, 1991, 1994) have focused specifically on proprioceptive representation but have used a memoryguided task in which the hand is replaced in a recently occupied location. The specific implications of these studies for proprioceptive representation are raised in the General Discussion section.

Many other studies have focused on V:P matching tasks under the name pointing or reaching, in order to investigate the sensorimotor transformation between extrinsic visual coordinates and intrinsic proprioceptive coordinates (e.g., Prablanc, Echallier, Komilis, \& Jeannerod, 1979; Rossetti, Tadary, \& Prablanc, 1994). Distortion of visual input by displacing prisms typically produces misreaching, which is gradually reduced by processes of recalibration and adaptation when perceptual information about errors is available (Redding \& Wallace, 1996). However, despite numerous investigations of sensorimotor transformation between modalities, relatively few studies have focused on the spatial representations underlying these transformations. In particular, few studies have reported matching errors at a range of locations in peripersonal space. The studies of Helms-Tillery et al. (1991,
1994) are notable exceptions. They studied the errors of 4 subjects attempting to replace the index finger at a previously occupied location in space and found large but idiosyncratic errors in performance.

We have therefore used a matching task to study the representation of egocentric space in normal adult human subjects, with particular emphasis on the proprioceptively perceived position of the hand. Matching errors were collected at a range of locations from left to right, spanning the midline. Furthermore, the conditions of matching were manipulated in our experiments to identify whether matching errors arose from inaccuracy in the perceived position of the target, or from inaccuracy in the parameters of the matching movement.

\section{EXPERIMENT 1}

Experiment 1 measured matching errors at nine target locations spanning the midline from left to right in the three matching conditions used by earlier studies (V:P, $V P: P$, and $P: P$ ) in 12 normal subjects.

\section{Method}

Procedure. The subjects were seated at a specially constructed table, made of wooden board $(1300 \times 800 \mathrm{~mm}$, and $15 \mathrm{~mm}$ thick $)$. Standard graph paper was fixed to the upper surface of the board, exactly aligned with the edge of the table. Nine points were marked on the graph paper with 1-mm crosses, extending across the subject's midline in a left-to-right direction. The targets were $70 \mathrm{~mm}$ apart, and they spanned a total $560-\mathrm{mm}$ range across the midline. This range of targets was chosen so that the subject could easily position the index finger of either hand on the extreme targets without movement of the torso. A line of masking tape was fixed over the subject's clothing, lying over the sternum on the body midline. The subject was positioned with this marker directly in front of the central target (Target 5) at a horizontal distance of $225 \mathrm{~mm}$ (see Figure 1). A cotton sheet was attached to the edge of the target board and to the subject's shoulder, to prevent any visual feedback of the movement of the arm under the table when matching target positions.

The subjects held a fine-tipped pen (Staedtler Lumocolor, model 315 ) in a tripod grip between the thumb, index finger, and middle finger. The subject was instructed to hold the pen as close as possible to the tip. On instruction to match a specified target location (see later), the subject moved the hand gripping the pen and made a single mark directly below where the target had been perceived to be located. A second sheet of graph paper was fixed to the underside of the target board, carefully aligned with the upper sheet. The location of each mark was measured with a ruler to generate the matching error on each trial in the $X$ (left-to-right) and $Y$ (nearbody-far-from-body) directions.

Conditions. The experiment was designed factorially with three within-subjects factors: target (nine levels, 1-9), matching condition (three levels, V:P, VP:P, and P:P), and the hand used for matching (two levels, left or right). The order of conditions was counterbalanced across subjects. The order of the matching hand factor was also counterbalanced within matching conditions, so that half the subjects always used the left hand to make matching judgments before the right hand, and the other half used the reverse order. A different random order of target positions was used for each subject in each combination of the condition and hand factors. Three trials were conducted at each level of condition, hand, and target factors. 


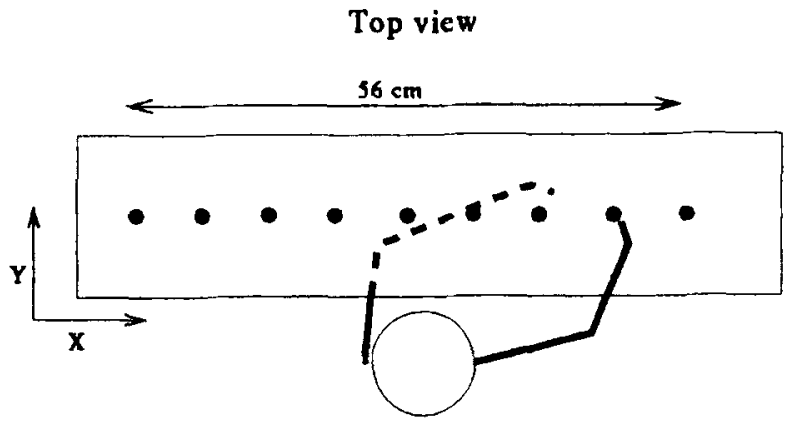

Front view

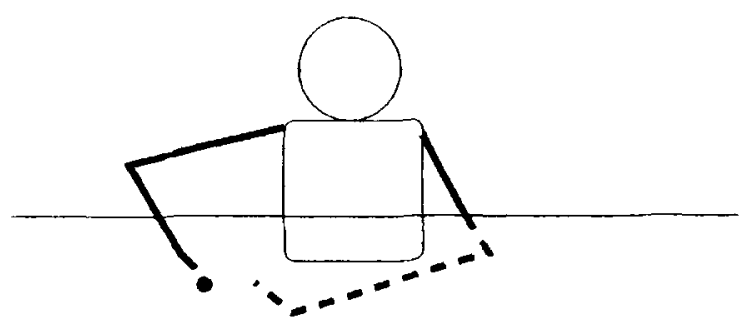

Figure 1. Apparatus used in Experiment 1. Note the hand placed on the target on the table surface (VP:P and P:P conditions only), and the other hand attempting to mark the table in the corresponding location underneath.

The order of trials was randomized, so that these three repeated trials were not successive.

Instructions. The instructions to the subjects varied with the matching condition. In the $V: P$ condition, the experimenter read aloud the number of a target between 1 and 9 , following a random sequence specific to that subject. These numbers corresponded to labels visible next to the targets. The subjects were instructed to use the pen gripped in the matching hand under the table to make a single mark exactly underneath the designated target and then return the matching hand to the rest position. The instructions emphasized to the subjects that they could pause and correct the position of the matching hand should they wish (i.e., the matching movements need not be ballistic). (In practice, the subjects tended to make the movements quite rapidly, and without obvious corrections, as observed in previous studies; see Baud-Bovy \& Viviani, 1998).

In the VP:P condition, the experimenter again read out the number of the designated target. The subjects held a compass tip in the "target hand" (i.e., whichever hand was not currently holding the matching pen under the table). They actively placed the compass tip on the designated target, and the experimenter verified its location. They then moved the matching hand under the table to mark the target location as before.

In the $\mathrm{P}: \mathrm{P}$ condition, the subjects again held the compass tip in the target hand. The experimenter passively moved the subjects' target hand to the designated target, precisely placing the pen tip on the target location. The subjects then performed the matching action under the table as before.

The subjects were instructed to return both the matching hand and the target hand to a rest position in their lap after each trial, and the experimenter verified that they did so. Finally, the subjects were instructed to remain still throughout the experiment and to avoid any movement of the head or torso at any time.
Subjects. Twelve subjects participated in the experiment. All were students at University College London, who volunteered their participation. Six subjects were male, and 6 were female. All subjects were strongly right-handed according to the Edinburgh Handedness Inventory (Oldfield, 1971). The age range was $18-25$ years (mean 20.9 years). All subjects had normal or corrected-to-normal vision, and none had any known neurological impairment.

\section{Results}

Interrater reliability. Matching errors were measured from the subject response sheets using a millimeter rule and measuring to the nearest millimeter. Since this measurement stage involves a subjective element, a subset of the data was measured independently by two of the experimenters, and the resulting measurements were compared. The intraclass correlation coefficient between the two raters' scores was 99.

Dependent variables. The constant and variable matching errors in the $X$ (left-right) and $Y$ (anterioposterior) directions for the three trials made by each subject at each target location in each matching condition with each matching hand were calculated (Figure 2). Since the number of matches in each condition was low, we used unbiased standard deviation estimators as measures of variable error. We divided the analysis into two stages. First, we performed a multivariate analysis of variance (MANOVA) with canonical discriminant analysis to identify how the pattern of errors varied across conditions and across targets. Whereas previous studies have decided a priori on a single composite measure of performance, such as absolute error, we preferred the MANOVA approach because it first identifies significant effects and then reveals which dependent variables, and thus which aspects of spatial representation, show the effects. Second, we performed univariate analyses where appropriate on those dependent variables shown by the MANOVA to be important.

Effects of matching condition. A significant MANOVA main effect of condition was observed $[F(8,38)$ $=6.609, p<.001$, Wilks's lambda approximation]. Canonical discriminant analysis showed that the first canonical variate accounted for $84 \%$ of the variance between the three conditions. The standardized canonical coefficients (SCCs; i.e., the extent to which each dependent variable contributed to the discriminant function, after taking into account the different scales of measurement for the dependent variables) are shown in Table 1. Inspection of each condition's scores on this variate showed that the greatest difference was between the P:P condition (score $=1.74, S D=0.40$ ) and both the V:P and VP:P conditions (scores $=1.27, S D=0.32$, and $0.99, S D=$ 0.27 , respectively), though all pairwise comparisons were significant beyond the .05 level in protected post hoc tests.

First, this result clearly replicates the findings of Wann (1991), von Hofsten and Rosblad (1988), and van Beers et al. (1996) regarding the ordering of matching performance in the three conditions. VP:P shows the best performance, followed by V:P, followed by P:P. Second, the 


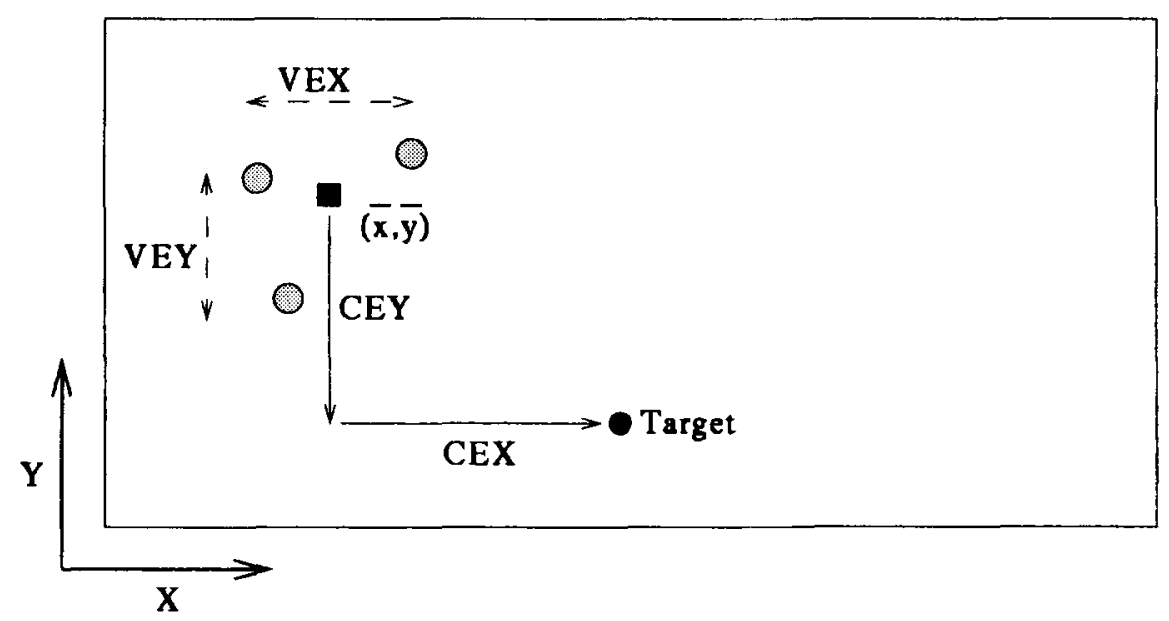

Figure 2. Calculation of dependent variables. The gray circles represent three attempts to match the target black circle. The means of the $X$ and $Y$ coordinates of the matching attempts gives the constant error, and their standard deviation gives the variable error. $C E X$, constant error in $X$ direction; CEY, constant error in $Y$ direction; $V E X$, variable error in $X$ direction; VEY, variable error in $Y$ direction.

use of discriminant analysis shows that the differences between these conditions are primarily in the variable error in the $X$ dimension. The fact that variable error is more effective in discriminating the conditions than constant error is consistent with the concept that the improved performance in the VP:P condition arises because integration of visual and proprioceptive representations provides a spatial map of greater precision (van Beers et al., 1996).

Effects of target position. Canonical discriminant analysis reveals a significant main effect of target position $[F(32,315)=4.557, p<.001$, Wilks's lambda approximation]. Only the first canonical variate had an eigenvalue greater than unity. It accounted for $61 \%$ of the intertarget variability. The SCCs for this variate are also given in Table 1. Note that the relative importance of each variable to intertarget variability is given by its absolute value rather than its signed value. The largest SCC is therefore that for constant error in the $Y$ dimension. This indicates that the feature of matching performance that changes most between left and right target positions is the degree of bias toward or away from the body. Figure 3 shows the scores of each target on the canonical variate (the axis has been inverted to allow comparison with Figure 5). This bias is expressed most strongly by targets close to the midline, which generally deviate away from the body, and is less strongly expressed by peripheral targets on both the left and the right. That is, the straight line of target locations is matched in a convex pattern bowed around the egocenter. Interestingly, this bias would be missed in tasks such as line bisection, in which the response is itself confined to the left-right axis. We will return later to this bias in univariate analyses of the constant error in $Y$.
Figure 3 also shows lower scores on the canonical variate (i.e., smaller errors) for extreme-left targets than for extreme-right targets. This indicates a far-left advantage in spatial representation used in matching tasks. A specific post hoc test of this finding was made by comparing the scores of Target 1 (far left) and Target 9 (far right) on the canonical variate. This test showed a significant far-left advantage $[F(1,11)=9.264, p=.011]$. Thus, we find no evidence for a far-left disadvantage in the spatial representations used in matching tasks, but we find significant support for an effect in the opposite direction.

Condition $\times$ target interaction. The effect of target position was found to interact significantly with condition $[F(64,680)=3.610, p<.001$, Wilks' lambda approximation]. The SCCs for the first and second canonical variate (explaining $48 \%$ and $41 \%$ of the interaction variance, respectively) are shown in Table 1, and the values of the variates for each condition and target combination are shown in Figure 4.

The P:P condition is clearly the source of the condition $\times$ target interaction for both the canonical variates.

Table 1

Standardized Canonical Coefficients from MANOVAs of Experiment 1

\begin{tabular}{lcccc}
\hline & \multicolumn{2}{c}{ Effect } & & \\
\cline { 2 - 4 } & Condition & Target & & \multicolumn{2}{c}{ Target $\times$ Condition } \\
\hline Variate number & 1 & 1 & 1 & 2 \\
Variance explained & $84 \%$ & $61 \%$ & $48 \%$ & $41 \%$ \\
Constant errors & & & & \\
$\quad X$ & -0.1339 & 0.6871 & 0.2667 & 1.4072 \\
$Y$ & 0.2043 & -1.1442 & 2.7870 & -0.358 \\
Variable errors & & & & \\
$X$ & 0.9485 & 0.3424 & 0.0600 & 0.0553 \\
$Y$ & -0.0776 & 0.6659 & 0.2968 & -0.119 \\
\hline
\end{tabular}




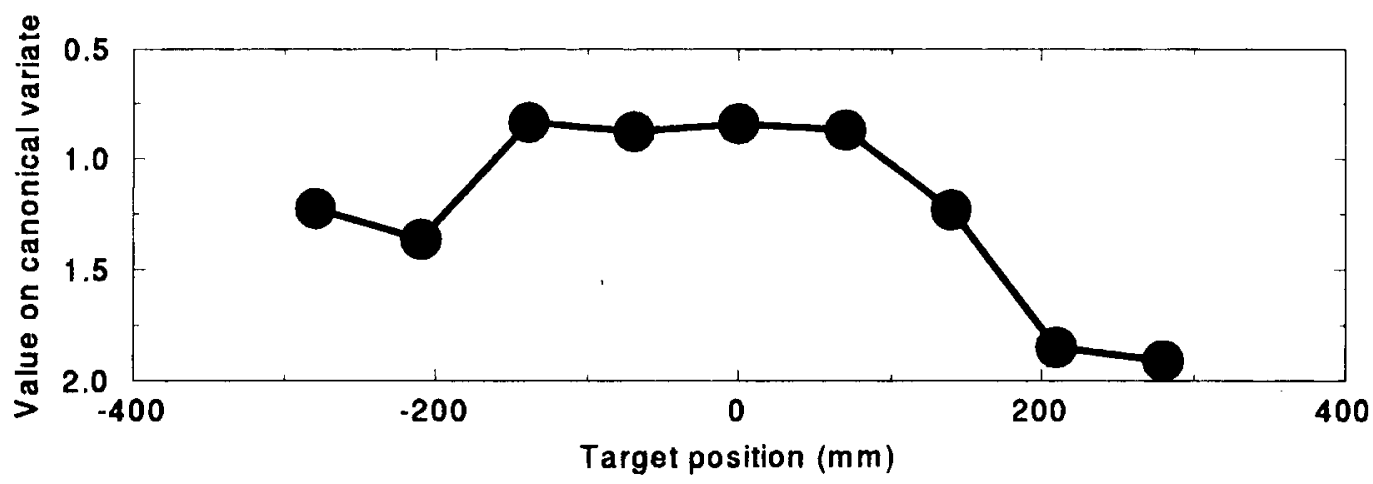

Figure 3. Score for each level of the target factor on the canonical variate best discriminating the target locations in Experiment 1. The ordinate has been inverted to facilitate comparison with Figure 5. Note the lower score indicating lower matching errors (higher value because of inverted ordinate) for far-left targets compared with far-right targets.

First, Figure 4A shows that the P:P does not exhibit the curvature of the left-right axis around the egocenter seen in the two visual conditions. Since this variate loads heavily on the constant error in $Y$, it seems that matching errors in the P:P condition are curved in the other direction, toward the body. The second canonical variate, which accounts for almost as much variance as the first, loads heavily on the constant error in $X$. Figure $4 \mathrm{~B}$ shows that, in the two visual conditions, this variate is expressed negatively for left-side targets and positively for rightside targets. Given the high loading on constant error in $X$, this feature appears to be a tendency to overshoot the target with the movement of the matching hand. That is, in the visual conditions, the matching hand was placed farther from the midline than it need be to match the target. This effect is absent, or even reversed, in the P:P condition, where the canonical variate is expressed positively for some targets on the left and negatively for some on the right. That is, in the P:P condition, subjects appear to undershoot the target, moving insufficiently far from the midline.

Univariate analyses. The canonical variates in Table 1 show that the main effect of target and its interaction with condition load mainly on the constant error measures rather than on the variable error measures. Since each variate appears to load quite specifically on either $X$ or $Y$ constant error, and since successive canonical variates are uncorrelated, we subjected the $X$ and $Y$ constant error data to independent univariate analyses to obtain a clearer picture of spatial matching. Figures 5, 6, and 7 indicate the average matching positions for each hand at the nine target conditions in the V:P, VP:P, and P:P conditions, respectively. The data have been presented separately according to the hand used (see later). The distance between the target location (diamond symbol) and the average matching location indicates the constant errors in $X$ and $Y$. Note that the ordinate scale has been enlarged to emphasize the constant error in the $Y$ dimension.
The predominant feature of the V:P and VP:P conditions is the pronounced bowing of the perceived target position away from the egocenter. This pattern has previously been observed in pointing experiments similar to our V:P matching paradigm (Prablanc et al., 1979). Two features of this bowing will be mentioned here. First, the effect is much more dramatic in the V:P condition than in the VP:P condition. Since the only difference between these conditions is the addition of proprioceptive information about the target location in the VP:P condition, this ordering suggests that the bowed pattern arises from a distortion in mapping visually perceived target locations to proprioceptive coordinates. The distortion would be reduced by the integration of a second, proprioceptive source of information into the mapping.

One possible interpretation attributes the bowing of space to the matching movements made under the table, rather than the perceived positions of the targets above the table. If movements in egocentric space, such as those made by the matching hand to the target position, were coded not in Cartesian space but in an egocentric space defined by coordinates of amplitude and direction from the egocenter (Ghilardi et al., 1995), then a bowing of space like that in Figure 5 might result from a range effect in motor programming. A tendency to make movements of the same amplitude irrespective of direction would lead to a curved set of matching positions like that shown. We have two reasons for rejecting a motor explanation of matching errors along these lines. First, the bowing effect is absent in the P:P condition, although it involved presumably similar matching movements. Second, we transformed the entire data set into egocentric polar coordinates centered on the projection of the sternum marker into the horizontal plane and analyzed the constant error of the distance $(R)$ from this egocenter to each matching location. This polar analysis revealed a significant bowing effect [seen in the main effect of target; $F(8,80)=$ $4.284, p<.001]$. This effect was due to a tendency for 

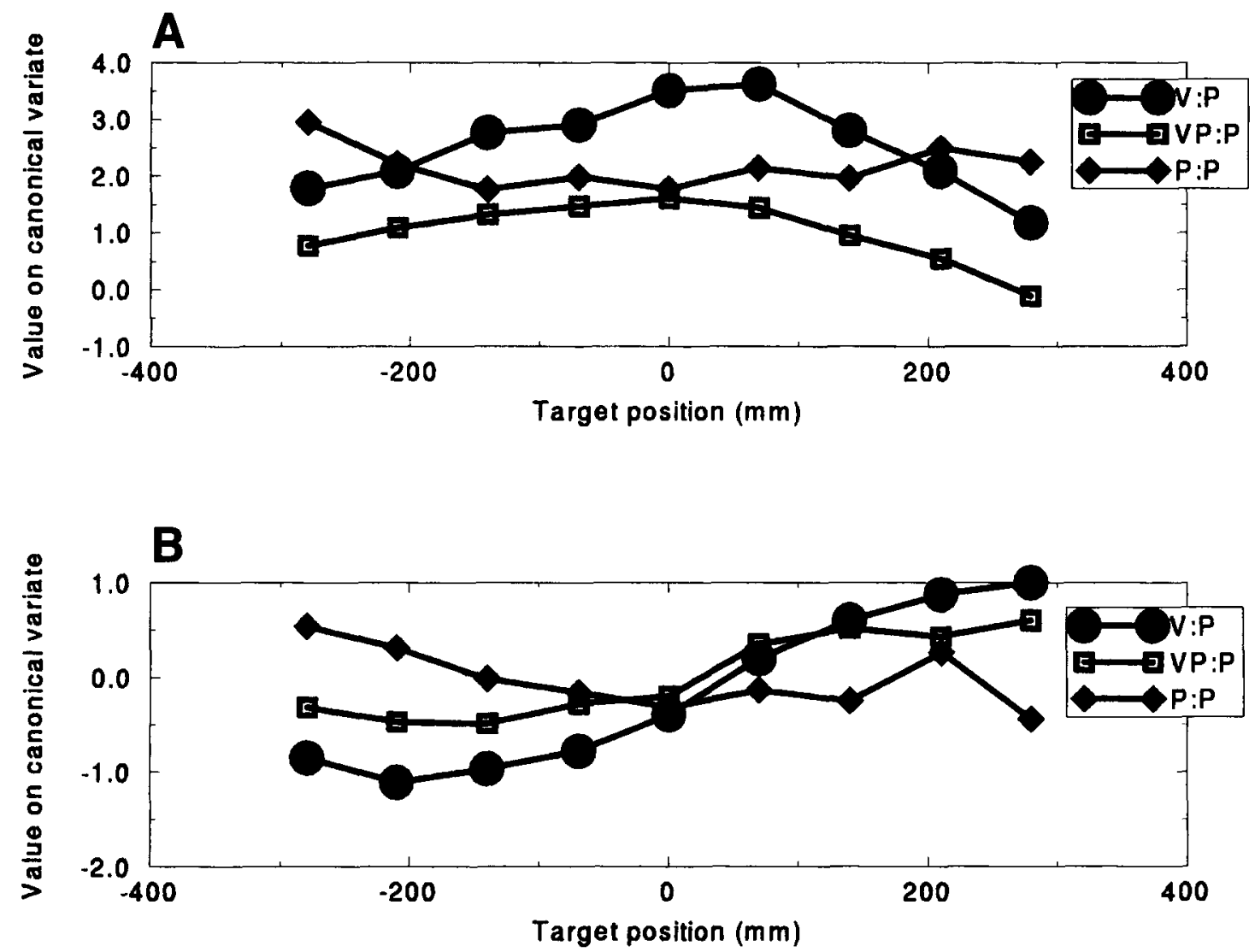

Figure 4. Score for each level of the target factor on the canonical variates best for the condition $\times$ target interaction in Experiment 1. (A) First canonical variate, explaining $48 \%$ of interaction variance, and loading primarily on $Y$ constant error. Note the bowing away from the egocenter for the V:P and VP:P conditions, but not for the P:P condition. (B) Second canonical variate, explaining $41 \%$ of interaction variance. Note the positive slope for the V:P and VP:P conditions, but not for the P:P condition.

vectors to midline targets to have larger amplitudes, precisely in the same way that those targets produce greater constant errors in $Y$ in our Cartesian analysis. Thus, the bowing cannot be due to our choice of Cartesian coordinates to study a system operating in polar coordinates.

Finally, the constant errors in the $X$ dimension showed a rightward bias across all conditions, though this did not approach statistical significance over subjects $[M=$ $3.95 \mathrm{~mm} ; t(11)=1.338, p=.208]$. Thus, our data do not lend support to accounts of a general leftward or rightward bias in egocentric representation. This finding contrasts with reports of significant rightward or leftward biases in the very different task of pointing to the subjective midline.

Hand bias. A second difference between the conditions is that the P:P condition shows a dramatic difference in the matching errors for the two hands. This "hand bias" is much less evident for VP:P and even smaller for V:P. Inspection of Figures 5 and 6 shows that the bowing of space is seen for both hands, as is the less salient tendency for eccentric targets to be matched too far from the midline in the $X$ dimension. The difference between the hands in P:P matching (Figure 7) does not resemble that in the other two conditions. No bowing effect is seen. Instead, the target effect clearly constitutes a positive slope when matching a left-hand target and shows an almost exactly opposite pattern when matching a right-hand target. This tendency for the hands to differ in slope is clear in the P:P condition, seemingly absent in the V:P condition, and appears to be present, though masked by the bowing effect, in the VP:P condition.

We fitted quadratic regressions to the constant error in $Y$ of each subject's data for each hand, using a quadratic term to model the bowing effect and a linear term to model the slope seen in Figures 5-7. We expressed the difference in the linear slopes for the two hands as a percentage of each subject's difference in the P:P condition. The V:P condition had a mean of $23 \%$ of the P:P condition's hand bias, and the VP:P condition had $36 \%$. That is, the hand bias in the VP:P condition was intermediate between those in the other two conditions.

Analysis of constant errors in $X$ for the P:P condition revealed a second component of hand bias, in addition to the dramatic slope difference. When the right hand was 


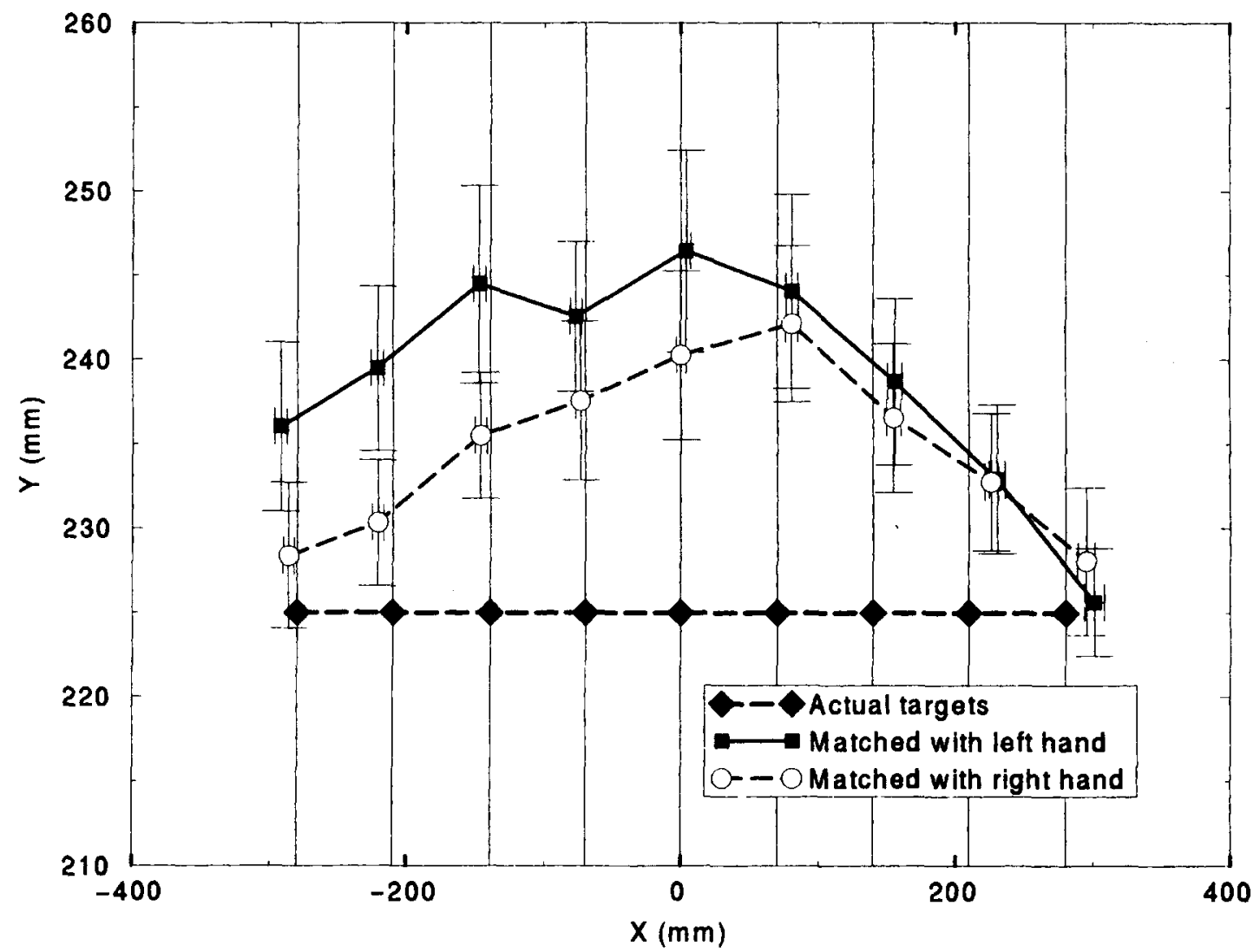

Figure 5. Constant errors in V:P matching. The ordinate scale is considerably magnified to show $Y$ constant errors. Note the bowing away from the egocenter, the tendency to overshoot extreme targets (rightward error for right targets, leftward error for left targets), and the similar patterns of error for the two hands. Error bars show standard errors over subjects in $X$ and $Y$ dimensions.

placed on the target location, the P:P matching positions chosen by the left hand were slightly displaced to the right of the actual target locations. An almost exact inverse of this effect was seen when indicating the perceived positions of the left hand by matching movements of the right hand under the target surface. In this case, constant errors in $X$ showed the perceived position of the left hand to be slightly to the left of the actual target.

In summary, there appears to be a bias in the perceived position of each hand in P:P matching. The bias can be described as a shift from the midline toward the shoulder of the target hand and a rotation of the frontoparallel plane in a clockwise direction for the right hand and a counterclockwise direction for the left hand. Statistically, these effects were highly significant. The shifts in the $X$ dimension $(15.6 \mathrm{~mm}$ rightward for the perceived position of the right hand and $8.2 \mathrm{~mm}$ leftward for the perceived position of the left hand) led to a significant main effect of hand on $X$ constant error $[F(1,11)=8.670, p=.013]$. Because the constant error in $Y$ seems to have a linear relation to target position (Figure 7), we predicted each subject's constant error in $Y$ from the target position in a linear regression and converted the mean slope of these regressions into an angle to describe the rotational bias shown by each hand in perceiving the frontoparallel plane. The mean angle of rotation was $3.9^{\circ}$ clockwise $\left(2.1^{\circ} S D\right)$ for the perceived position of the right hand and $3.7^{\circ}$ counterclockwise $\left(2.3^{\circ} S D\right)$ for the perceived position of the left hand. In both cases, the mean of the subjects' slopes differed from 0 at the .001 level on Student's $t$ tests. While we have analyzed the translational and rotational elements of the error pattern independently, we note that, in principle, both types of error might arise from a single rotation, with an unknown center of rotation some distance from the midline.

\section{Discussion}

The P:P condition shows a hand bias quite different from that of the V:P and VP:P conditions. This bias appears to consist of two independent geometric elements that can be conveniently described in Cartesian space. First, the target hand above the surface is always perceived to be displaced in the frontoparallel plane slightly in the direction given by its shoulder of origin. Because our targets were positioned quite centrally in peripersonal space, and because we did not precisely measure shoulder position in our subjects, our data do not reveal whether targets positioned beyond the shoulder location would be 


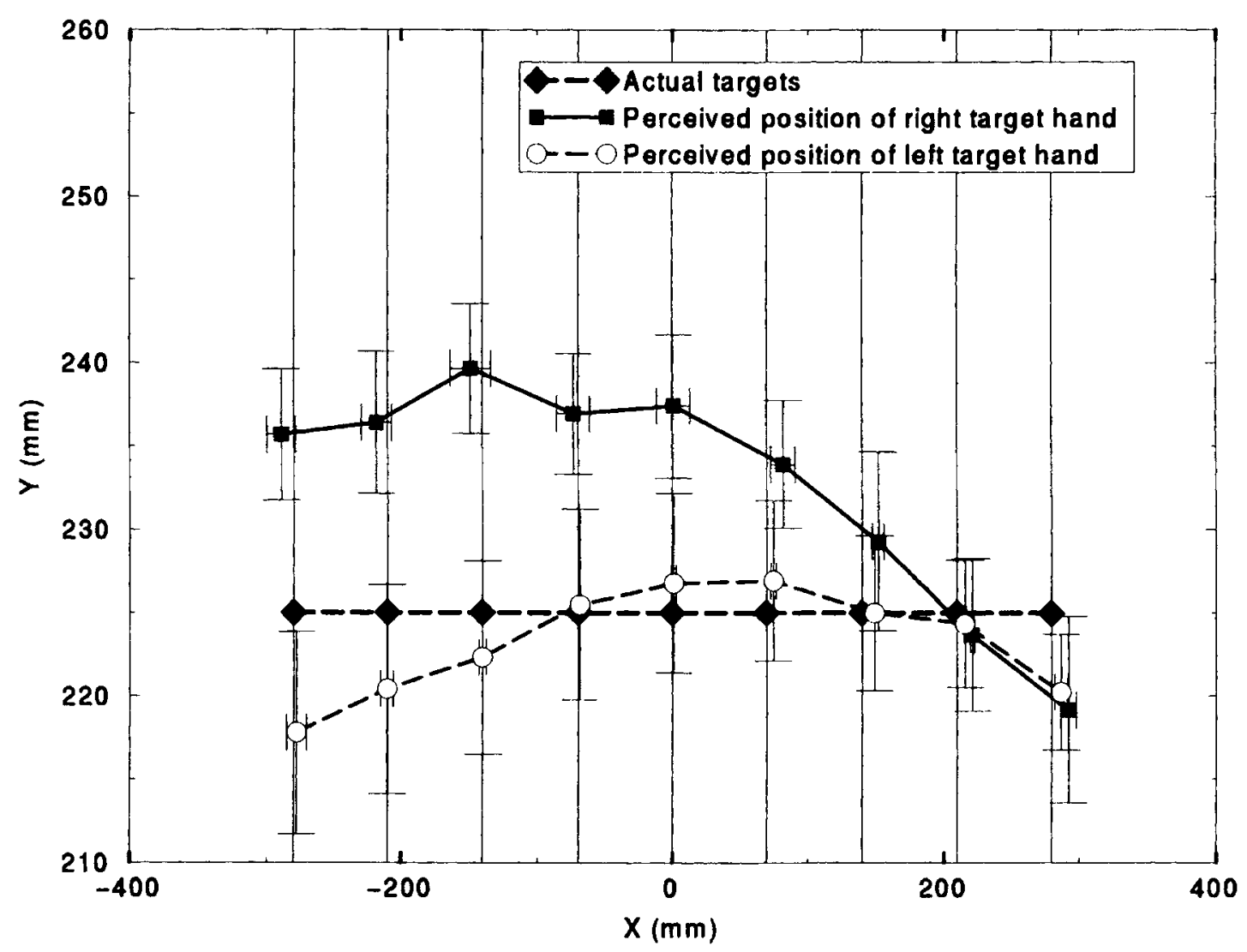

Figure 6. Constant errors in VP:P matching. Error bars show standard errors over subjects in $X$ and $Y$ dimensions.

displaced back toward the shoulder and the midline or whether they show translation in the same direction as our more centrally positioned targets. Second, the frontoparallel plane of the target is perceived to be angled slightly so as to point toward the shoulder of origin.

This bias appears not to have been reported previously. Wann and Ibrahim (1992) have reported a drift in the perceived position of the limb. However, this drift probably cannot explain the present findings for two reasons. First, the drift begins only after $15 \mathrm{sec}$ without visual or proprioceptive cues regarding hand location: The matching movements of our subjects were considerably faster. Second, their proprioceptive drift was directed toward the body, whereas our hand bias effect produced errors in the opposite direction at some target locations (Figure 7).

Where does this hand bias come from? An important initial question concerns whether it arises from a distortion of the proprioceptively perceived position of the hand above the table or from a motor error in the matching movements made by the hand under the table. We believe the significant reduction in hand bias in the other visual conditions indicates that the bias is largely representational rather than motor in origin.

We assume the matching movements made under the surface were similar in all three conditions, though we did not measure kinematics. Any hand bias of motor origin should have been seen in the V:P and VP:P conditions as well as in the P:P condition, yet this was not the case. By elimination, it seems plausible that this finding reflects a bias in the represented position of each hand. This hypothesis was tested directly by manipulating the perceptual information available for proprioceptive target representation in Experiment 2.

The results of Experiment 1. suggest that the representation of proprioceptive space measured in the P:P condition is quite different from the representation of visual space measured in the V:P condition. In particular, there appears to be no single unified representation of proprioceptive space. Instead, each hand appears to operate in its own egocentric space, with the two hands exhibiting almost mirror-image distortions in opposite directions. How can these distortions be explained? Our data are consistent with the possibility that each hand maintains its own representation of egocentric space that uses a frame of reference based not on the egocenter but on the motor workspace of that hand. Since the origin for most hand positioning movements is the shoulder, it seems plausible that hand position is represented in a frame of reference shifted somewhat toward the shoulder and using a principal axis biased toward the tangent around the shoulder- 


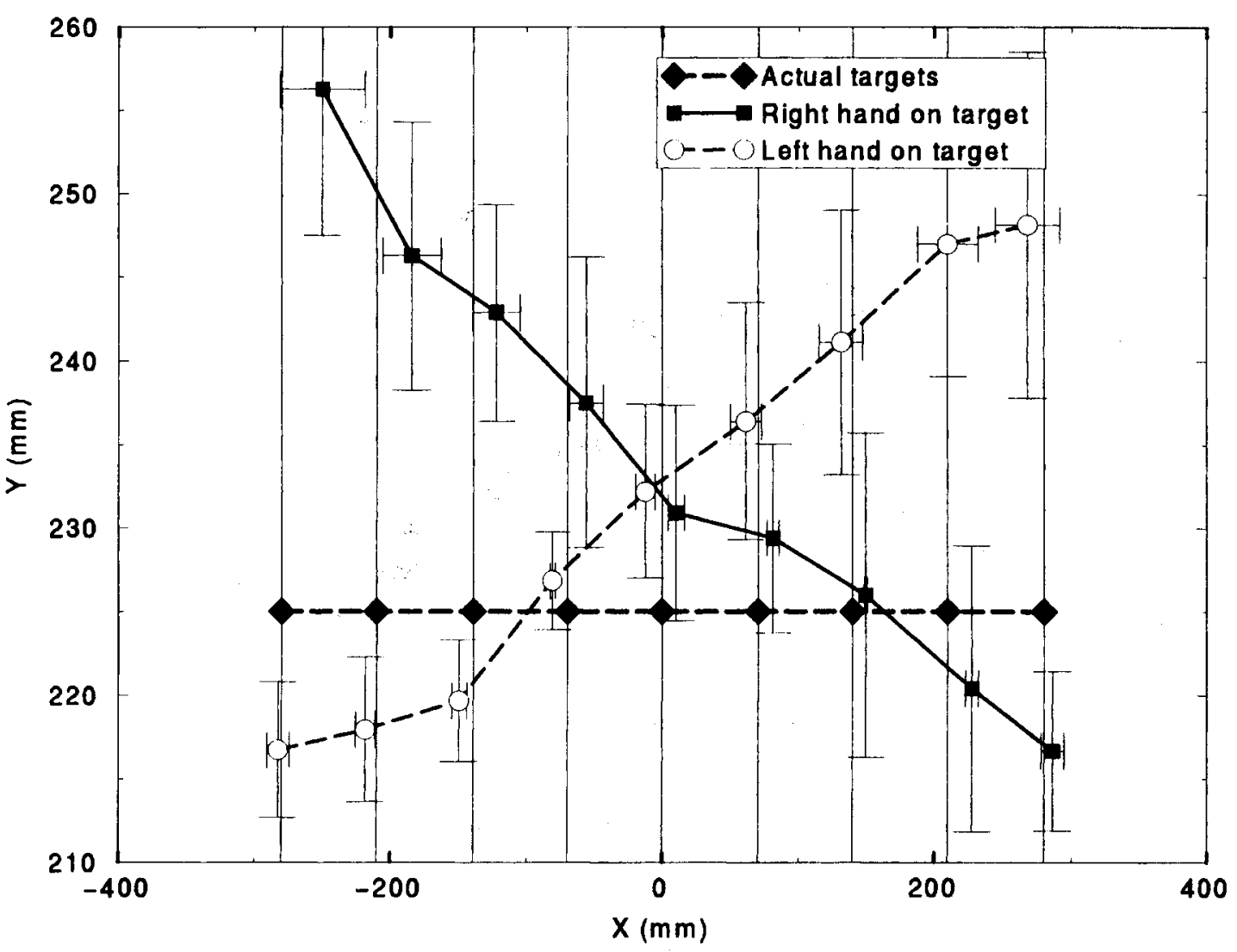

Figure 7. Constant errors in P:P matching. Note the hand bias, revealed in the different slopes and sideways shifts of left and right target hands. Error bars show standard errors over subjects in $X$ and $Y$ dimensions.

that is, toward the reachable workspace of the arm. In this sense, our finding suggests a functional rather than absolute representation of the perceived position of the hand in egocentric space. We note that earlier studies using prism adaptation (Prablanc, Tzavaras, \& Jeannerod, 1975) have also suggested separate spatial representations for each arm. Prablanc et al. (1975) were able to induce opposite adaptive shifts on each arm by separate exposures to prisms of opposite sign, suggesting that a sensorimotor adaptation need not apply throughout peripersonal space but could be learned separately for each arm.

\section{EXPERIMENT 2}

We have already asked whether the hand bias in P:P matching is perceptual or motor. One class of evidence for errors in perceptual representation of proprioceptive target positions would come from manipulations of the perceptual experience that subjects have of the target on the top of the matching surface. If manipulated experience of the target leads to differences in hand bias, then the hand bias must be perceptual rather than motor in origin, since the matching movements made under the table are presumably unaffected by the manipulated perceptual experience.

Experiment 2 therefore contrasted P:P matching in a condition in which subjects' hands were passively placed in the target position with a condition in which subjects actively moved to the target location by haptic guidance.

\section{Method}

The passive condition was designed to replicate the P:P condition of Experiment 1 precisely. In the active condition, in contrast, a small drill bit was embedded in a hole in the target surface at each target location, so that the drill bit just protruded above the target surface. This tactile indication of the target location allowed the subjects to locate the target themselves on each trial by active haptic exploration beginning from a starting position in their lap. The target hand was actively returned to this starting position in between trials. The experiment thus used a factorial design with three conditions: matching condition (active or passive), matching hand (left or right), and target position (1-9). The geometrical arrangement of the targets, the randomization of targets over trials, the structure of each matching trial, the instructions to subjects, and the calculation of dependent variables were as in Experiment 1 . The matching condition was counterbalanced over subjects, so that half matched active targets first followed by passive targets, and the other half performed the conditions in the reverse order. The order of matching hands was similarly counterbalanced within each matching condition. 


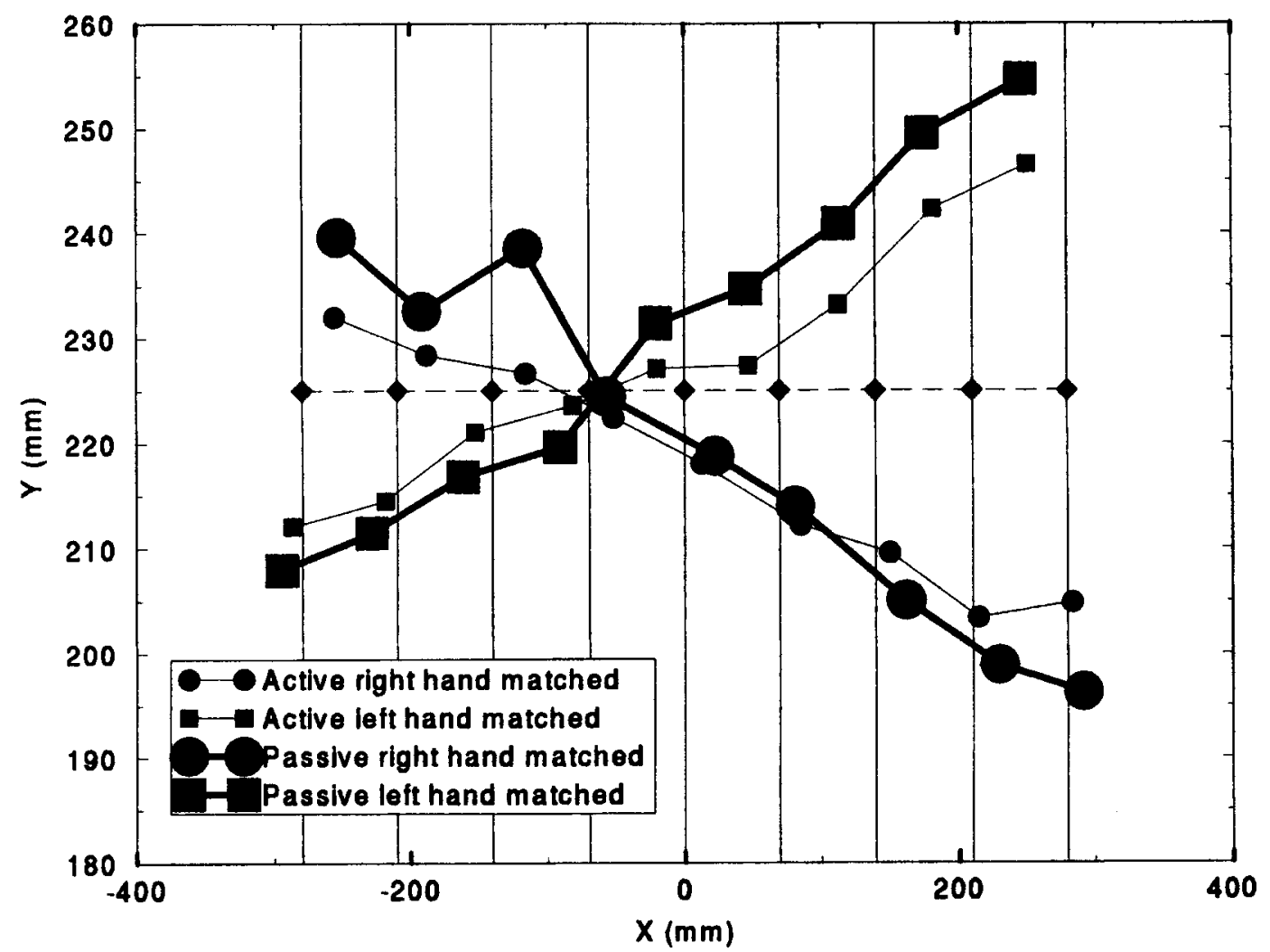

Figure 8. Hand bias for active versus passive placement of target hand in P:P matching in Experiment 2. Note reduced slopes in the active condition.

Six subjects participated in the experiment. All were males (age range, 17-19 years) recruited from a local further education college. All were strongly right-handed according to the Edinburgh Handedness Inventory (Oldfield, 1971), had normal or corrected-tonormal vision, and had no known neurological disorders.

\section{Results}

The matching positions for each combination of condition, hand, and target position are shown in Figure 8. The results were analyzed statistically using three-way repeated measures analyses of variance (ANOVAs) on constant error in $X$ and in $Y$, with condition, hand, and target position as the within-subjects factors.

First, the hand bias of Experiment 1 was replicated in Experiment 2. Thus, in the translational component of the bias, the perceived position of the right hand was $16.5 \mathrm{~mm}$ to the right of its actual location, and the perceived position of the left hand was $21.5 \mathrm{~mm}$ to the left of its actual location. This led to a significant main effect of hand on the constant error in $X[F(1,5)=12.207, p=.017]$. No other effects or interactions were significant. Turning to constant error in $Y$, Figure 8 shows an interaction of hand with target location, replicating the diagonal bias seen in Experiment 1 . This effect was statistically significant $[F(8,40)=18.171, p<.001]$. Furthermore, the three-way interaction between matching condition, hand, and target was significant $[F(8,40)=11.868, p=.001]$. This arose because the hand $X$ target interaction was less pronounced in the active condition than in the passive condition. We also computed the angles of the rotational bias, using the same procedure as in Experiment 1 . The mean values are shown in Table 2. An ANOVA on these angles revealed a significant effect of hand $[F(1,5)=20.579, p=.006]$ and a significant hand $\times$ condition interaction $[F(1,5)=$ $30.761, p=.003]$. This last effect is shown in Figure 8, where the patterns of constant error for active targets are clearly less exaggerated than those for passively located targets. The angles of rotation for active targets were on average $65 \%$ of those for passive targets.

\section{Discussion}

The hand bias in P:P matching is reduced when subjects actively move their hand to the target location rela-

Table 2

Mean Angle (in Degrees) Between the Line of Targets and the Line of Perceived Target Locations from Experiment 2

\begin{tabular}{lcrc}
\hline Condition & Target Hand & $M$ & $S D$ (across subjects) \\
\hline Active & Left & 3.4 & 2.5 \\
Active & Right & -3.1 & 1.8 \\
Passive & Left & 4.9 & 2.7 \\
Passive & Right & -4.8 & 2.9 \\
\hline
\end{tabular}


tive to when they are passively positioned at that location (cf. Paillard \& Brouchon, 1968). In fact, some active muscle contraction probably occurred in the "passive" condition because the arm was not supported; however, since we did not measure EMG, we cannot be sure how much. Therefore, the effect reported here is presumably only partial. However, it is statistically highly significant and in the same direction seen in other studies (Paillard \& Brouchon, 1968). Experiment 2 therefore showed that the subject's perceptual experience of the target position influences the matching error. This finding suggests that matching errors arise from perceptual or representational factors operating on the perceived position of the target hand. Since the movement of the matching hand under the table was presumably comparable in active and passive conditions, motor factors seem to have a lesser influence.

The results of Experiment 2 suggest that the hand bias arises from the frame of reference for proprioceptive representation. Additional involvement of the efferent components of position sense, which are available to subjects in the active condition but should be absent or minimal in the passive condition, reduces this bias significantly. Since we do not know the exact proportions in which efferent and proprioceptive mechanisms contribute to position sense, we cannot exclude some degree of hand bias in the efferent system.

\section{EXPERIMENT 3}

Our goal in Experiment 3 was to investigate the generality in hand bias in P:P matching seen in Experiments 1 and 2 and to investigate whether the hand bias was indeed attributable to use of a lateralized effector-specific representation of the target hand. We also tested whether manual dominance influenced the relative sizes of righthand and left-hand biases.

\section{Method}

Twelve new subjects participated in Experiment 3. These were divided into four groups each of 3 subjects on the basis of gender and Edinburgh Handedness Inventory (Oldfield, 1971) scores. The groups were male right-handers, male left-handers, female righthanders, and female left-handers. The right-handed and left-handed subjects all reported consistent preference of the right or left hand for all the items in the Edinburgh Handedness Inventory, except for 1 male left-handed subject, who reported preferential use of the right hand with scissors and toothbrush, despite using the left hand for writing and other unimanual tasks.

In Experiments 1 and 2, the targets were always located on the upper surface, and the matching movements were always made under the surface. Therefore, we cannot exclude the possibility that the hand bias arises because of the different arm configurations used to place the fingertip in the target position above and below the table. To confirm that the hand bias is a genuine feature of the perceived position of the target hand rather than an artifact of the limb configurations used, Experiment 3 added an additional factor of target surface with two levels: over and under. The target-over condition replicated the arrangement in Experiment 1, in which the subject's hand was passively placed on the target location above the table, and the matching movement was made under the table. In the target-under condition, the reverse arrangement was used - that is, the experimenter passively positioned the subject's hand on target positions located under the table, and the subject made matching movements above the table to match the target position.

Experiment 3 also used the subject's nose as one component of the matching task, in order to investigate whether the bias in P:P matching was indeed due to use of lateralized representations of hand position. The effectors used for matching depended on the level of the target surface factor, so the design was not fully factorial. In the target-over condition, the effector positioned on the target could be the left hand, the right hand, or the nose. When the target was the left or right hand, the matching movement under the table was made with the other hand. When the target was the nose, we included two conditions: matching the nose position with the left hand and matching the nose position with the right hand. The matching conditions were necessarily different for the case in which the target was under the table, because a seated subject cannot place the nose on the underside of the table. In this case, the target effector was either the left or the right hand. For each target hand, matching movements were made above the table both with the left hand or the right hand (according to condition) and with the nose. Matching with the nose could not be measured accurately and will not be discussed here.

In Experiment 3, only three target positions were used. These corresponded to the midline and to points $164 \mathrm{~mm}$ to the left and right of the midline. The target axis was located $116 \mathrm{~mm}$ from the edge of the table in the frontoparallel plane. A sternum marker was aligned with the midline target, as in Experiment 1 . The distance from the edge of the table to the subject could not be fixed, because larger subjects needed to sit farther away from the table in order to reach the targets with the nose. Likewise, the reduced left-right range of target positions was necessary due to the much more limited range of positions that can be reached by the nose of a seated subject, relative to the hands.

The instructions to the subjects and the trial structure were generally similar to those in the P:P condition of Experiment 1, with a few modifications. A piece of masking tape was placed on the tip of the subject's nose, and a thin crosshair was drawn on the tip of the nose exactly on the midline. In conditions in which the nose served as a target for matching, the experimenter held the subject's head and pushed the subject's head downward from the resting seated position, passively flexing the subject's hips and neck so as to precisely position the nose crosshair on the target marked on the upper surface. To make finger and nose matching comparable, the subjects in Experiment 3 placed the tips of their index fingers on targets, rather than holding a compass tip as in Experiments 1 and 2. Furthermore, the finger or the nose remained fixed on the target location while the subject made two attempts to match its position.

\section{Results}

The analysis of this experiment is presented in two distinct ways, according to the hypotheses tested. First, we report on the hand biases when matching one hand with the other. Second, we compare matching errors for hand targets with matching errors for nose targets.

Hand matching. In these analyses, we investigated (1) whether hand bias in P:P matching occurred for targets both above and below the target surface and (2) whether left- and right-handers displayed similar hand biases. Nose-matching conditions were excluded from these analyses.

Since the hand bias is primarily seen in the constant error in the $Y$ dimension, we performed a five-way repeated measures ANOVA on this dependent variable. Gender and manual dominance were two between-subjects 


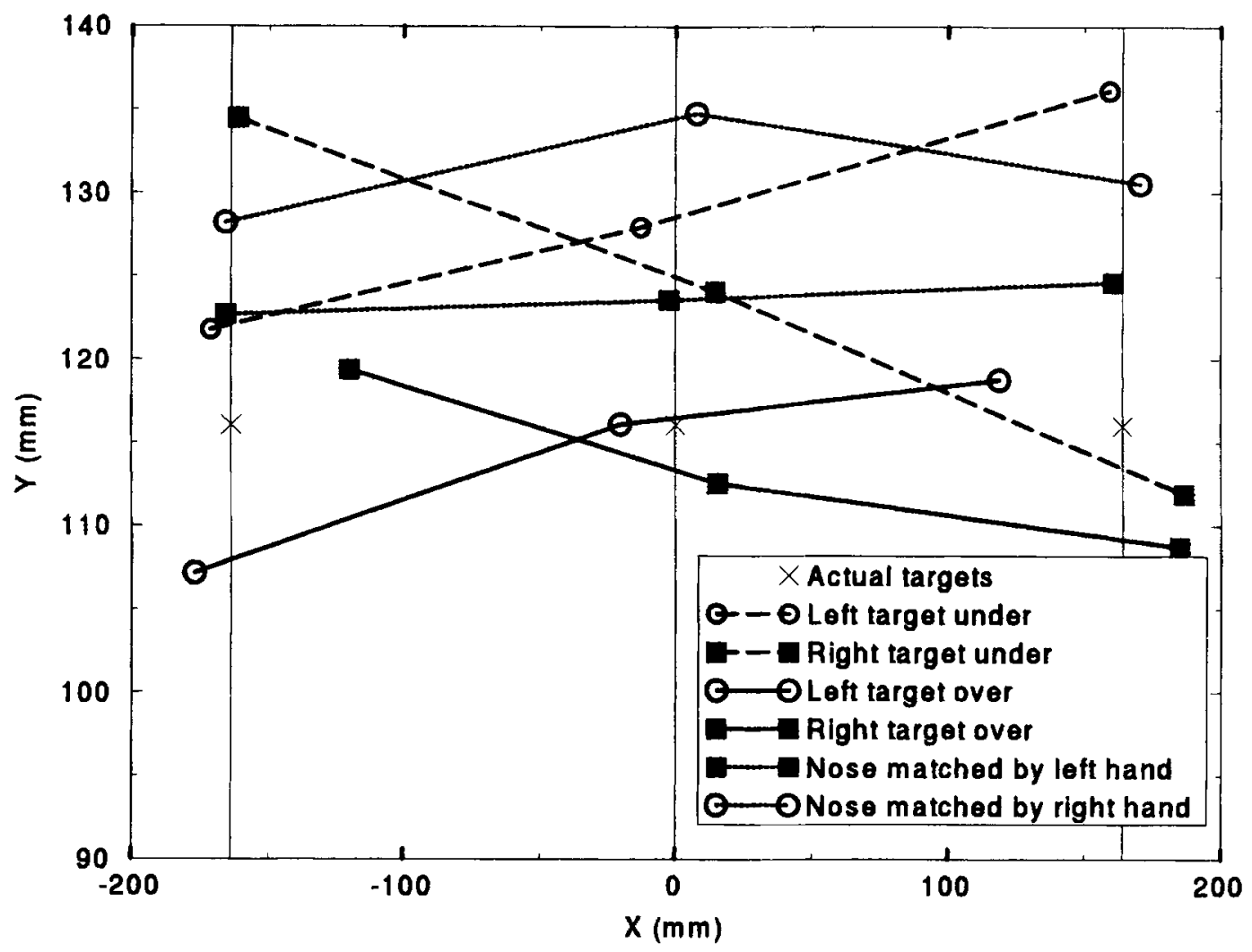

Figure 9. Hand bias in Experiment 3. Note comparable slopes and sideways shifts for targets above (solid lines) and below (dashed lines) the table. Also note the absence of hand bias when matching the nose with the left or right hand (dotted lines).

factors; target surface (over or under), target hand (left or right), and target position (left, midline, or right) were three within-subjects factors. The three-way interaction of target surface $\times$ target hand $\times$ target position was not significant $[F(2,16)=2.369, p=.126]$. In other words, the pattern of hand bias observed did not reliably vary with the matching surface used. This is illustrated by the comparable crossover interactions for solid (target above) and dashed (target underneath) lines of Figure 9. The results showed a highly significant interaction of target hand with target position $[F(2,16)=10.787, p=.001]$, replicating the diagonal hand bias seen in Experiments 1 and 2. This effect is shown graphically in Figure 9.

Analysis of the $X$ constant error also replicated the translational hand bias found in Experiments 1 and 2. The main effect of target hand was significant $[F(1,8)=11.022$, $p=.011]$. The effect was in the same direction for targets above and beneath the table, as shown in Figure 9, although the interaction of target hand and target surface showed a trend toward significance $[F(1,8)=3.653, p=.092]$.

Interestingly, some effects of handedness were found in P:P matching. First, we found a significant main effect of handedness on constant error in $Y$, with right-handers showing larger errors than left-handers $[6.9 \mathrm{~mm}$ vs. $0.7 \mathrm{~mm}$, respectively; $F(1,8)=5.548, p=.046]$. Furthermore, we found a significant interaction between manual dominance and gender, whereby the difference between left- and right-handers was more dramatic for females than for males [left-right difference, $-12.2 \mathrm{~mm}$ for females, $0.0 \mathrm{~mm}$ for males; $F(1,8)=5.473, p=.047]$. No significant interactions between handedness and target hand were found, suggesting no effects of manual dominance.

Drift analysis. In Experiment 3, unlike in Experiments 1 and 2, the subjects made multiple attempts to match a target effector remaining in a static location. This procedure introduces the possibility of a drift across time in the perceived position of the target hand (cf. Wann \& Ibrahim, 1992). We therefore measured the drift in $X$ and $Y$ values between the first and second matching attempts in each condition, and subjected the drift measures to a three-way (target surface $\times$ target hand $\times$ target position) ANOVA. The mean drifts did not differ significantly from $0(-0.9 \mathrm{~mm}$ in $X,-0.2 \mathrm{~mm}$ in $Y ; p>.5)$. Two factors significantly affected $X$ drift. First, the difference between a $-3.8-\mathrm{mm}$ leftward shift for targets under the table, and a $2.0-\mathrm{mm}$ shift for targets above the table just achieved statistical significance $[F(1,11)=4.847, p=.05]$. Second, the target surface $\times$ target hand interaction was significant $[F(1,11)=7.012, p=.023]$ due to the surface effect being almost entirely confined to right-hand targets. Drift in the $\mathrm{Y}$ dimension revealed a significant interaction only between target surface and target position 
$[F(2,22)=3.708, p=.041]$ due to an enhanced proprioceptive drift toward the body in the case of targets under the table in right hemispace. These analyses suggest that proprioceptive drift between successive matching attempts at the same target were not consistent across target locations and choice of target hand.

Nose matching. We now turn to the second analysis of Experiment 3-namely, a comparison of matching nose targets with matching hand targets. Our previous results suggested that the hand-bias effect in P:P matching arose from a tendency to represent the perceived position of each hand in a frame of reference biased toward its shoulder of origin. That is, hand position is represented in an arm-centered space rather than an egocentric space. A clear prediction of this account would be an absence of hand bias when matching a midline target, such as the nose. We therefore constructed a repeated measures ANOVA to address this hypothesis. Since the nose of a seated subject could be placed on target positions only on the upper side of the target surface, only data with targets on the upper surface were included in this analysis. The within-subjects factors in the analysis were target part (nose or hand), matching hand (left or right), and target position (left, midline, or right). The three-way interaction between target part, matching hand, and target position was highly significant for $Y$ constant error $[F(4,32)=$ $5.528, p=.002]$. The perceived position of the nose when matched with the left and right hands is shown by the dotted traces in Figure 9. Inspection of Figure 9 shows the origins of this interaction. The familiar hand bias clearly exists when matching a target hand but not when matching the nose. Post hoc testing of appropriate pairs of conditions showed that the right-hand and left-hand biases were each significantly reduced in the nose-matching condition. More importantly, the nose-matching condition showed no significant interaction between the hand used to match the nose and the target position for $Y$ constant error $[F(2,16)=0.178$, n.s. $]$. Thus, we found no evidence for a diagonalizing hand bias in the nose-matching condition. Likewise, analysis of the $X$ constant error produced no evidence for a translational bias in nose matching $[F(2,16)=0.206$, n.s. $]$.

The results of Experiment 3, therefore, indicate that the hand bias in P:P matching is not an artifact of the specific limb configurations used to test it in Experiment 1. Rather, it appears to depend on the hand position, irrespective of the side of the target surface (and, thus, of the limb configuration) used. Second, this effect disappears when using the hands to match a midline structure, such as the nose. Again, we assume that the matching movements were similar in both cases. Therefore, the most likely explanation for the hand biases seen in all three experiments is the use of an arm-specific frame of reference for the perceived position of the hand in space.

\section{GENERAL DISCUSSION}

We have presented three experiments on spatial representation in which (1) the mapping of subjective space across the midline from left to right has been described in detail, (2) important differences in the structure of visual and proprioceptive space have been revealed, and (3) a clear hand bias in the proprioceptive representation of hand position has been identified, whereby the hand is perceived as translated and rotated toward its shoulder of origin. We now discuss some specific psychological issues raised by these results.

The most salient feature of all three experiments is a consistent hand bias in P:P matching. In this effect, the right hand is perceived to be translated slightly toward the right shoulder and rotated clockwise, whereas the left hand shows the opposite translational and rotational biases. The center of rotation appears to lie, on average, in the midsaggital plane. Our experiments have excluded the possibility that this arises entirely from an artifact of the arm configurations used in our matching and have also shown that it reflects a perceptual bias in the representation of the position of the target hand rather than any bias in either the perception or movement of the matching hand. However, analysis of Experiment 1 showed that a residual $23 \%$ of the P:P hand effect remained present even in V:P matching. Since the V:P condition has a visual, and not proprioceptive, target, this residual hand bias cannot be due to the perceived position of the target hand. We therefore assume that it reflects some residual hand bias occurring in the matching movements made under the table. On this reading, it is perhaps surprising that the perceived position of the target hand above the table shows four times the bias of an identical hand performing proprioceptively guided movements under the table. One possible explanation of this asymmetry might be that the brain processes proprioceptive information about target locations in a different way from the proprioceptive information received from the moving limb.

A second interesting feature of the hand bias is the apparent mirror symmetry of its effects on the left and right hands. Both translational and rotational components seem remarkably similar in size for the two hands, though opposite in sign. We found no evidence that either effect is more pronounced for the dominant hand than for the nondominant hand. This symmetry in hand bias recalls the anatomical symmetry of the two hands and arms themselves around the body midline. It is consistent with the possibility that hand bias arises from the use of a proprioceptive frame of reference for hand position that is influenced by the shoulder of origin. On this reading, the perceived position of the hand is biased toward its shoulder of origin. Furthermore, it is rotationally biased into the primary axis of its movement, since the actions of the left hand primarily rely on elbow and shoulder flexions directing it toward the midline, and vice versa for the right hand. These biases could be interpreted as basing the perceived position of each hand within a distinct egocentric frame of reference appropriate for its normal action.

Biases in the proprioceptively perceived position of the hand have been reported previously, notably by HelmsTillery et al. (1991, 1994) and by Baud-Bovy and Viviani (1998). As mentioned before, those studies all involved 
returning to a previously remembered position. Those studies produced higher errors (see Baud-Bovy \& Viviani, 1998 , p. 1541) than the P:P matching errors shown in Figure 9. This difference may reflect a substantial contribution of spatial memory decay. Baud-Bovy and Viviani reported a leftward shift when the right hand is used for both locating targets and subsequent pointing (their RR condition), and they reported a rightward shift when the left hand is used for locating and the right hand for pointing (LR condition). In our terms, their right targets were perceived to the left of their true location, and their left targets were perceived to the right-the opposite of the translational bias in our P:P matching data. This apparent contradiction seems difficult to explain. However, the memory component may play a role. A simple perceptual or representational bias would not produce any shift in the $R R$ condition, since any bias would apply both during target location and during subsequent pointing. Second, the translational errors in their Figure 2 vary across the left-right axis, whereas ours do not. Third, their $X$ shift is roughly twice as large as that reported in our Experiment 1 . In future research, we plan to compare matching and memory-guided tasks in the same target locations in the same subjects, using a range of memory-holding periods, to investigate whether memory affects on proprioceptive representation.

In their studies, Helms-Tillery et al. (1991, 1994), again using a memory-guided procedure, found large and idiosyncratic errors in a switched-limb condition, though they used fewer subjects than we used in the experiments reported here. Their focus was to determine the coordinate system used for proprioceptive information about hand position. They concluded that this information "is most readily utilized when it is represented in arm orientation parameters" (Helms-Tillery et al., 1991, p. 777). In contrast, our hand-bias effect can be represented very simply as a translation and rotation in Cartesian coordinates. However, we have not calculated a comparable hand bias in arm orientation coordinates. Interestingly, HelmsTillery et al. (1991) also required a simulation to exclude the possibility that subjects simply reproduce the angles of each participating joint to reattain the memorized target. That strategy would attain the target position without need for a truly spatial representation of hand position. In P:P matching, this strategy is impossible, because matching joint angles of the two arms does not match the positions of the two hands, except in the single case of midline targets. A joint-matching strategy would therefore predict better matching in the midline than elsewhere. Our Figure 4, however, shows very similar amounts of error for targets $140 \mathrm{~mm}$ to either side of the midline, providing experimental evidence that joint matching is unlikely.

Conclusive proof that the hand biases measured here reflect partly shoulder-based frames of reference is hard to obtain by traditional psychological methods of selec- tive influence: The position of the shoulder cannot be perturbed without also affecting the ability of the hand to perform matching tasks. Corroborative evidence for a contribution of shoulder-based representation might, however, come from studying individuals of different anthropometric types. In particular, the distance of the shoulder center of rotation from the body midline varies considerably across individuals. Thus, the translational components of the hand bias in proprioceptive representation might be proportional to the distance of the shoulder from the midline. This hypothesis predicts a positive correlation between the size of the hand-bias effect and the breadth of the shoulders.

A final question concerning the hand bias is whether it depends on the anatomy of the body or on the functional motor experience of the subject. Thus, for example, would a change in the balance of use of the two hands (e.g., when one arm is immobilized following fracture) increase the hand bias in the intact arm while reducing it in the unused arm? Neurophysiological studies have shown that cortical representation of the body surface is not static. Indeed, the size of the cortical territory representing a given portion of the body fluctuates according to the relative use of that body part and its neighbors (Recanzone, Merzenich, Jenkins, Grajski, \& Dinse, 1992). The changes in the amount of cortical representation do not necessarily imply biases in the origin or frame of reference of that representation, but the possibility that biases are related to cortical plasticity cannot be ruled out. In future research, we plan to investigate whether hand bias can be modulated by the specific proprioceptive experiences and motor training of normal human subjects in a laboratory setting.

\section{REFERENCES}

Baud-Bovy, G., \& Viviani, P. (1998). Pointing to kinesthetic targets in space. Journal of Neuroscience, 18, 1528-1545.

BISIACH, E. (1993). Mental representation in unilateral neglect and related disorders. Quarterly Journal of Experimental Psychology, 46A. 435-461.

Bowers, D., \& Heilman, K. M. (1980). Pseudoneglect: Effect of hemispace on a tactile line bisection task. Neuropsychologia, 18, 49l-498. Bradshaw, J. L., Nettleton, N. C., Nathan, G., \& Wilson, L. (1983). Head and body space to left and right, front and rear: II. Visuotactual and kinesthetic studies and left-side underestimation. Neuropsychologia, 21, 475-486.

GhIlardi, M. F., Gordon, J., \& GHez, C. (1995). Learning a visuomotor transformation in a local area of work space produces directional biases in other areas. Journal of Neurophysiology, 73, 2535-2539.

HeAd, H. (1920). Studies in neurology. London: Hodder \& Stoughton. Helms-Tillery, S. I., Flanders, M., \& Soechting, J. F. (1991). A coordinate system for the synthesis of visual and kinesthetic information. Journal of Neuroscience, 11, 770-778.

Helms-Tillery, S. I., Flanders, M., \& Soechting, J. F. (1994). Errors in kinesthetic transformations for hand apposition. NeuroReport, 6 , 177-181.

JEANNEROD, M. (1988). The neural and behavioural organisation of goal-directed movements. Oxford: Oxford University Press.

OLDFIELD, R. C. (1971). The assessment and analysis of handedness: The Edinburgh Inventory. Neuropsychologia, 9,97-113.

PAILLARD, J. (1991). Brain and space. Oxford: Oxford University Press. 
Paillard, J., \& Brouchon, M. (1968). Active and passive movement in the calibration of position sense. In S. J. (Ed.), The neuropsychology of spatially oriented behavior (pp. 35-55). Homewood, IL: Dorsey.

Pierson-Savage, J. M., \& Bradshaw, J. L. (1987). Mapping of extracorporeal space by vibrotactile reaction times: A far-left-side disadvantage. Perception, 16, 283-290.

Prablanc, C., Echallier, J. F., Komilis, E., \& Jeannerod, M. (1979). Optimal response of eye and hand motor systems in pointing at a visual target. I. Spatio-temporal characteristics of eye and hand movements and their relationships when varying the amount of visual information. Biological Cybernetics, 35, 113-124.

Prablanc, C., Tzavaras, A., \& Jeannerod, M. (1975). Adaptation of the two arms to opposite prism displacements. Quarterly Journal of Experimental Psychology, 27, 667-671.

Recanzone, G. H., Merzenich, M. M., Jenkins, W. M., Grajski, K. A., \& DiNSE, H. R. (1992). Topographic reorganization of the hand representation in cortical area $3 \mathrm{~b}$ of owl monkeys trained in a frequency-discrimination task. Journal of Neurophysiology, 67, $1031-1056$.

REDding, G. M., \& WaLlaCE, B. (1996). Adaptive spatial alignment and strategic perceptual-motor control. Journal of Experimental Psychology: Human Perception \& Performance, 22, 379-394.
Rossetti, Y., Tadary, B., \& Prablanc, C. (1994). Optimal contributions of head and eye positions to spatial accuracy in man tested by visually directed pointing. Experimental Brain Research, 97, 487 496.

van Beers, R. J., Sittig, A. C., \& Denier van der Gon, J. (1996). How humans combine simultaneous proprioceptive and visual position information. Experimental Brain Research, 111, 253-261.

von Hofsten, C., \& RosBlaD, B. (1988). The integration of sensory information in the development of precise manual pointing. Neuropsychologia, 26, 805-821.

WANN, J. P. (1991). The integrity of visual-proprioceptive mapping in cerebral palsy. Neuropsychologia, 29, 1095-2106.

WANN, J. P., \& IBRAhim, S. F. ( 1992). Does limb proprioception drift? Experimental Brain Research, 91, 162-166.

WERnER, H., WAPNER, S., \& BrUELl, J. H. (1953). Experiments on sensory-tonic field theory of perception: VI. Effect of position of head, eyes and of object on position of the apparent median plane. Journal of Experimental Psychology, 46, 293-299.

(Manuscript received June 16, 1997; revision accepted for publication October 20, 1998.) 